Barber, M. (1953). J. gen. Microbiol. 8, 104-110.

\title{
The Effect of Serial Passage in other Antibiotics on Penicillinase-producing Staphylococci
}

\author{
By MARY BARBER \\ Department of Bacteriology, St Thomas's Hospital Medical School, London
}

SUMMARY: Seven strains of penicillinase-producing Staphylococcus aureus (coagulase-positive) were serially transferred on streptomycin ditchplates; four of the seven streptomycin-resistant variants showed a decreased ability to produce penicillinase. In three instances this loss in penicillinase production was caused by a decreased growth rate. The fourth strain was unstable in relation to penicillin, and exposure to streptomycin appeared to select the naturally occurring penicillin-sensitive variants.

Three of the seven strains were similarly exposed to chloramphenicol; of the three chloramphenicol-resistant variants one was slightly more resistant to penicillin, one considerably less resistant and one unchanged in relation to penicillin. The increased resistance to penicillin was associated with an increase in growth rate. The strain showing a decrease in penicillin-resistance was unstable, and chloramphenicol selected out the naturally occurring penicillin-sensitive variants.

Recent investigations at the Johns Hopkins University (Chandler, Davidson, Long \& Monnier, 1951; Monnier \& Schoenbach, 1951) have indicated that exposure of penicillin-inactivating bacteria to other antibiotics leads to a reduction in their capacity to produce penicillinase. Monnier \& Schoenbach (1951) studied three penicillin-destroying bacteria, one strain each of Staphylococcus aureus, Klebsiella pneumoniae and Bacterium aerogenes. After serial transfer in media containing aureomycin, chloramphenicol, terramycin or streptomycin these organisms showed a 2- to 64-fold increase in sensitivity to penicillin, and this was associated with a loss in penicillinase production. Subsequent transfer of the antibiotic-resistant variants in media free from antibiotics did not lead to a return of their former ability to destroy penicillin. The present investigation was undertaken to study this phenomenon more fully and to see whether it was associated with any other changes.

\section{METHODS}

The organisms used were seven strains of coagulase-positive staphylococci, all of which produced penicillinase. Two of the strains (D3 and STH10), both belonging to phage-group $6 / 47$, had been kept in the laboratory for more than a year; five had been recently isolated from the noses of nurses in a maternity department and their phage-types were $3 \mathrm{~A}$ (strain W187), 3B/3C (strain W120), 47/54 (strain W135R), 52A (strain W 305) and non-typable (strain W21).

Serial transfer in the presence of antibiotics was carried out by means of ditchplates. The medium used was $1 \%$ 'Lab-Lemco', $1 \%$ peptone, $0.5 \% \mathrm{NaCl}$ and $2 \%$ Bacto shred agar. Ditches were cut at one side of the plate 
and filled with the same medium containing 100-1000 $\mu \mathrm{g}$. streptomycin $/ \mathrm{ml}$. or $100 \mu \mathrm{g}$. chloramphenicol $/ \mathrm{ml}$. The cultures were plated out at right angles to the ditch and subcultures made from the edge of growth nearest to the ditch. Control cultures were plated on ordinary nutrient agar plates without antibiotic. In the case of streptomycin subcultures were made at 1- to 2-day intervals. With chloramphenicol, at first subcultures were made at similar intervals, but it was later found that resistance to this antibiotic occurred more quickly if the interval was prolonged and subcultures were made after 6-7 days incubation.

Sensitivity to antibiotics was estimated before and after passage in streptomycin or chloramphenicol by the serial dilution method. The medium was Lab-Lemco broth and a large inoculum of 1-6 million bacteria was used. The results were read after $24 \mathrm{hr}$. incubation at $37^{\circ}$. All the parent strains were inhibited by from 2.5 to $10 \mu \mathrm{g}$. streptomycin $/ \mathrm{ml}$., except for strain W305 which was inhibited by $50 \mu \mathrm{g} . / \mathrm{ml}$, and all were inhibited by from 10 to $25 \mu \mathrm{g}$. chloramphenicol $/ \mathrm{ml}$. As regards penicillin, strains D3, STH10 and W 21 all grew readily in more than $250 \mathrm{u}$./ml.; strains W 120 and W 305 grew in $50 \mathrm{u} . / \mathrm{ml}$.; strains $\mathrm{W} 135 \mathrm{R}$ and $\mathrm{W} 187 \mathrm{grew}$ in $5-10 \mathrm{u} . / \mathrm{ml}$. The penicillinsensitive strains showed a sensitivity similar to that of the Oxford staphylococcus.

Penicillinase production was measured by the cylinder plate method, using the technique described in a previous paper (Barber, 1947).

Bacteria were counted by the rapid viable counting technique of Miles \& Misra (1938).

\section{RESULTS}

\section{Exposure to streptomycin}

Effect on sensitivity to streptomycin and pencillin. The seven penicillindestroying strains of Staph. aureus were serially subcultured on streptomycin ditch plates. The results of such exposure with regard to streptomycin and penicillin sensitivity are recorded in Table 1. It will be seen that in all strains there was a gross increase in resistance to streptomycin and that four strains

Table 1. Effect of exposure to streptomycin on sensitivity to

(a) streptomycin and $(b)$ penicillin

$\begin{array}{lccc}\text { Strain } & \begin{array}{c}\text { Number of } \\ \text { subcultures in } \\ \text { streptomycin }\end{array} & \begin{array}{c}\text { Streptomycin } \\ \text { increase }\end{array} & \begin{array}{c}\text { Penicillin } \\ \text { decrease }\end{array} \\ \text { D3 } & 12 & >10,000 \text {-fold } & \begin{array}{r}\text { Change in resistance } \\ \text { STH 10 }\end{array} \\ \text { W 21, Exp. 1 } & 16 & >10,000 & \text { No change } \\ \text { W21, Exp. 2 } & 16 & >10,000 & 10 \\ \text { W21, Exp. 3 } & 10 & 4,000 & >1,000 \\ \text { W120 } & 10 & 1,000 & 5 \\ \text { W187 } & 12 & >10,000 & \text { No change } \\ \text { W305 } & 7 & 1,000 & 2 \\ \text { W135R } & 7 & 100 & 2 \cdot 5 \\ & 4 & 2,000 & \text { No change }\end{array}$


showed a decrease in resistance to penicillin. This decrease was shown to result from a diminished ability to produce penicillinase.

Effect on growth rate. The four streptomycin-resistant variants with a lower resistance to penicillin than the parent cultures from which they were derived were investigated for other changes. It was found that they also differed from the parent cultures in rate of growth. In strains D3 and W 305 the difference was so great that it was readily seen by naked eye inspection of overnight cultures on nutrient agar plates (see Pl. 1, fig. 1). Thus the parent culture showed typical Staph. aureus colonies c. 1-2 mm. in diameter, whereas the colonies of the streptomycin-resistant variant were only pin-point in size. When broth cultures inoculated with a known number of organisms were counted at various intervals after incubation it was found, with each of the four strains, that the streptomycin-resistant variant multiplied at a significantly slower rate than the parent culture. The results are given in Table $\mathbf{2}$, from which it will be seen that the greatest difference in growth rate usually occurred during the first $3 \mathrm{hr}$. incubation. It seems also certain that, in the case of strains D3, STH10 and W187, the difference in growth rate between parent culture and streptomycin-resistant variant would be quite sufficient to account for a lower rate of penicillinase production leading to the 2- to 10-fold decrease in penicillin-resistance noted with these strains.

\section{Table 2. Rate of multiplication of parent cultures and streptomycin-resistant variants}

Increase in population

\begin{tabular}{|c|c|c|c|c|}
\hline \multirow[b]{2}{*}{ Strain } & \multicolumn{2}{|c|}{ After $3 \mathrm{hr}$. incubation } & \multicolumn{2}{|c|}{ After $24 \mathrm{hr}$. incubation } \\
\hline & $\begin{array}{l}\text { Parent culture } \\
\text { subcultured on } \\
\text { plain agar }\end{array}$ & $\begin{array}{l}\text { Streptomycin- } \\
\text { resistant } \\
\text { variant }\end{array}$ & Parent culture & $\begin{array}{l}\text { Streptomycin- } \\
\text { resistant } \\
\text { variant }\end{array}$ \\
\hline D3 & $15 \cdot 9 \times$ & $\mathbf{2 \cdot 3} \times$ & - & - \\
\hline W21 & $19 \cdot 9 \times$ & $7 \times$ & $170 \times$ & $170 \times$ \\
\hline W 187 & - & - & $148 \times$ & $84 \times$ \\
\hline W305 & $19 \times$ & $3 \times$ & $422 \times$ & $244 \times$ \\
\hline
\end{tabular}

Stability of strains in relation to penicillin resistance. Many strains of penicillinase-producing staphylococci are known to be unstable in that they yield a proportion of variants which are sensitive to penicillin, having lost their capacity to destroy the antibiotic (Barber, 1949). The incidence of such variants in parent and streptomycin-resistant cultures was investigated. The cultures were plated out thinly on plain agar plates and fifty colonies from each streaked across penicillin ditch plates. The results are given in Table 3.

Strains D3 and W305 did not show penicillin-sensitive colonies, whether or not they had been exposed to streptomycin. Penicillin-sensitive colonies were not isolated from the streptomycin-resistant variant of strain W187, but the parent strain W187 subcultured in the absence of streptomycin yielded three penicillin-sensitive colonies out of fifty tested. Such a proportion of penicillin-sensitive variants, however, would be masked by the penicillinase 
of the resistant colonies and have little effect on the penicillin sensitivity of the whole culture (Barber, 1949).

It will be seen that strain $\mathbf{W} 21$ is unstable in relation to penicillin, and that after each of three series of transfers in streptomycin the streptomycinresistant culture yielded a much larger proportion of penicillin-sensitive colonies than did the parent when subcultured in the absence of streptomycin. In Exp. 2, where the streptomycin-resistant variant showed $>1000$-fold

Table 3. Incidence of penicillin-sensitive variants after exposure to streptomycin

No. of penicillin-sensitive variant colonies (fifty tested)

$\begin{array}{lcc}\text { Strain } & \begin{array}{c}\text { Parent culture Streptomycin- } \\ \text { subcultured on } \\ \text { plain agar }\end{array} & \begin{array}{c}\text { resistant } \\ \text { variant }\end{array} \\ \text { D3 } & 0 & 0 \\ \text { W21, Exp. 1 } & 1 & 43 \\ \text { W21, Exp. 2 } & 4 & 50 \\ \text { W21, Exp. 3 } & 2 & 12 \\ \text { W187 } & 3 & 0 \\ \text { W305 } & 0 & 0\end{array}$

decrease in resistance to penicillin, penicillin-resistant colonies were not isolated even when the whole culture was plated out on a penicillin ditch plate. In Exps. 1 and 3, where the decrease in resistance to penicillin was 10- and 5-fold, the streptomycin-resistant variant yielded forty-three and twelve penicillin-sensitive colonies respectively. The cultures of strain W21 not exposed to penicillin only yielded one to four penicillin-sensitive colonies. A typical penicillin ditch plate, showing streaks of penicillin-resistant and sensitive colonies from strain W 21, is shown in Pl. 1, fig. 2.

When a penicillin-sensitive and penicillin-resistant colony from the parent culture W21 were serially transferred on streptomycin ditch plates it was found that the penicillin-sensitive variant developed resistance to streptomycin much more quickly than did the penicillin-resistant culture, and after nine subcultures became completely dependent on the antibiotic (see Pl. 1, fig. 3). It thus seemed probable that streptomycin did not increase the incidence of penicillin-sensitive variants from strain W21, but tended to select out those that were naturally occurring.

Stability of streptomycin-resistant variants. Most of the streptomycinresistant variants remained resistant to streptomycin and showed no change in growth rate after many transfers in broth without antibiotic. The poorly growing streptomycin-resistant variant of strain D3, however, after eight subcultures in the absence of antibiotic, yielded a culture which grew as luxuriantly as the parent strain and showed a 50-fold decrease in resistance to streptomycin. This moderately streptomycin-resistant variant was as resistant to penicillin as the parent culture, although the poorly growing and highly streptomycin-resistant variant had been 10 times less resistant to penicillin. 


\section{Exposure to chloramphenicol}

Effect on sensitivity to chloramphenicol and penicillin. Three strains were serially subcultured on chloramphenicol ditch plates and then tested for a change in sensitivity to chloramphenicol and penicillin. The results are shown in Table 4. All three strains showed some increase in resistance to chloramphenicol, but the degree was not great. In the case of strain W21, after nine subcultures in chloramphenicol there was a 5 -fold decrease in resistance to penicillin and after twelve subcultures the decrease was 1000-fold. In the case of strain W120 there was no change in resistance to penicillin after exposure to chloramphenicol. In the case of strain D3 the chloramphenicolresistant variant showed a 2 -fold increase in resistance to penicillin.

Table 4. Effect of exposure to chloramphenicol on sensitivity to

(a) chloramphenicol and (b) penicillin

\begin{tabular}{|c|c|c|c|}
\hline \multirow[b]{2}{*}{ Strain } & \multirow[b]{2}{*}{$\begin{array}{c}\text { Number of } \\
\text { subcultures in } \\
\text { chloramphenicol }\end{array}$} & \multicolumn{2}{|c|}{ Change in resistance } \\
\hline & & $\begin{array}{l}\text { Chloramphenicol } \\
\text { increase }\end{array}$ & $\begin{array}{l}\text { Penicillin } \\
\text { decrease }\end{array}$ \\
\hline D3 & 16 & 4-fold & 2-fold increase \\
\hline W21 & $\mathbf{9}$ & 2 & $\mathbf{5}$ \\
\hline W21 & 12 & 10 & 1000 \\
\hline W 120 & 16 & 4 & No change \\
\hline
\end{tabular}

Effect on growth rate. The growth rates of the parent and chloramphenicolresistant cultures in broth were compared after 3-5 hr. incubation at $37^{\circ}$. The results are given in Table 5 . It will be seen that the chloramphenicol-resistant variant of strain D3 multiplied somewhat more rapidly than did the parent culture, which probably accounted for the increase in resistance to penicillin. With the other strains there was no significant difference in growth rate between parent and chloramphenicol-resistant cultures.

Table 5. Rate of multiplication of parent cultures and chloramphenicol-resistant variants

$\begin{array}{lcc}\text { Strain } & \overbrace{\begin{array}{c}\text { Parent culture } \\ \text { subcultured on } \\ \text { plain agar }\end{array}}^{\begin{array}{c}\text { Increase in population after } \\ \mathbf{3 - 5} \mathrm{hr} \text {. incubation }\end{array}} & \begin{array}{c}\text { resistant } \\ \text { variant }\end{array} \\ \text { D3 } & 40 \times & 50 \times \\ \text { W21 } & 17 \times & 15 \times \\ \text { W120 } & 13 \times & 11 \times\end{array}$

Stability of strains in relation to penicillin resistance. Fifty colonies from each culture were streaked across penicillin ditch plates. The incidence of penicillinsensitive variant colonies is recorded in Table 6. Strains D3 and W120 did not yield any penicillin-sensitive colonies, whether or not they had been exposed to chloramphenicol. 
Table 6. Incidence of penicillin-sensitive variants after exposure to chloramphenicol

\begin{tabular}{|c|c|c|}
\hline & \multicolumn{2}{|c|}{$\begin{array}{l}\text { No. of penicillin-sensitive variant } \\
\text { colonies (fifty tested) }\end{array}$} \\
\hline Strain & $\begin{array}{c}\text { Parent culture } \\
\text { subcultured on } \\
\text { plain agar }\end{array}$ & $\begin{array}{l}\text { Chloramphenicol- } \\
\text { resistant } \\
\text { variant }\end{array}$ \\
\hline D3 & $\mathbf{0}$ & 0 \\
\hline W21, 9 subcultures & 2 & 46 \\
\hline W 21, 12 subcultures & 3 & 50 \\
\hline W 120 & $\mathbf{0}$ & 0 \\
\hline
\end{tabular}

Strain W 21 was again seen to be unstable in relation to penicillin sensitivity. After nine transfers in the presence of chloramphenicol, forty-six of fifty colonies tested were sensitive to penicillin; after twelve transfers all fifty colonies were penicillin-sensitive, although a few resistant colonies were isolated when a heavy inoculum of the whole culture was plated out on a penicillin ditch plate. After cultivation on nutrient agar without chloramphenicol only two to three penicillin-sensitive colonies were isolated.

\section{DISCUSSION}

When penicillinase-producing staphylococci are exposed to streptomycin or chloramphenicol some, but not all, strains show a variable change in their ability to produce penicillinase. The change is usually a decrease, but in one instance was a slight increase. It seems clear, however, from the results here recorded that this is not due to a specific action on penicillinase production, but results from one or both of two associated phenomena. First, the streptomycin- or chloramphenicol-resistant variant may multiply at a different rate from the parent. Secondly, if the original strain is unstable in relation to penicillin, exposure to streptomycin or chloramphenicol may tend to select the naturally occurring penicillin-sensitive variants.

The close association of growth rate with penicillinase production is clearly shown in relation to strain D3. The poorly growing streptomycin-resistant variant of this strain was unstable and reverted to a culture which grew as well as the parent and was only moderately resistant to streptomycin. The parent and the moderately streptomycin-resistant culture were highly and similarly resistant to penicillin, the poorly growing variant showed a 10-fold decrease in resistance to penicillin. Further, when this strain was exposed to chloramphenicol, it yielded a culture which multiplied more rapidly than the parent and showed a slight but definite increase in resistance to penicillin.

The incidence of penicillin-sensitive variant colonies from penicillininactivating strains of Staph. aureus was studied by Barber (1949) who showed that with some strains variants were few or none, whereas others yield a considerable proportion. Apart from repeated subculture Barber did not find any method of increasing the incidence of these naturally occurring variants. In the present investigation strain W21 was unstable in this respect, and a larger 
proportion of penicillin-sensitive variant colonies was isolated after passage in the presence of streptomycin or chloramphenicol than after passage in the absence of antibiotics. When penicillin-sensitive and penicillin-resistant colonies from the parent culture were passaged separately in the presence of streptomycin the penicillin-sensitive culture 'developed' resistance to streptomycin much more quickly than did the penicillin-resistant culture. It seems probable, therefore, that, at any rate with streptomycin, the antibiotic acted by selecting penicillin-sensitive variants, without affecting their actual incidence.

\section{REFERENCES}

Barber, M. (1947). Coagulase-positive staphylococci resistant to penicillin. J. Path. Bact. 59, 373.

BARBER, M. (1949). The incidence of penicillin-sensitive variant colonies in penicillinase-producing strains of Staphylococcus pyogenes. J. gen. Microbiol. 3, 274.

Chandler, C. A., Davidson, V. Z., Long, P. H. \& Monnier, J. J. (1951). Studies on resistance of staphylococci to penicillin: the production of penicillinase and its inhibition by the action of aureomycin. Johns Hopk. Hosp. Bull. 89, 81.

Miles, A. A. \& Misra, S. S. (1938). The estimation of the bactericidal power of the blood. J. Hyg., Camb. 38, 732.

Monnier, J. J. \& Schoenbach, E. B. (1951). The resultant sensitivity of microorganisms to various antibiotics after induced resistance to each of these agents. Antibiotics \& Chemotherapy, $1,472$.

\section{EXPLANATION OF PLATE}

Fig. 1. Strain D3. Parent culture and streptomycin-resistant variant.

Fig. 2. Strain W21. Penicillin-sensitive and penicillin-resistant variants streaked across a penicillin ditch plate.

Fig. 3. Strain W21. Penicillin-resistant $(R)$ and penicillin-sensitive $(S)$ variants plated out on a streptomycin ditch plate. Penicillin-sensitive culture shows complete dependence on streptomycin and growth of penicillin-resistant culture is favoured by streptomycin.

(Received 9 July 1952) 
Journal of General Microbiology, Vol. 8. No. 1
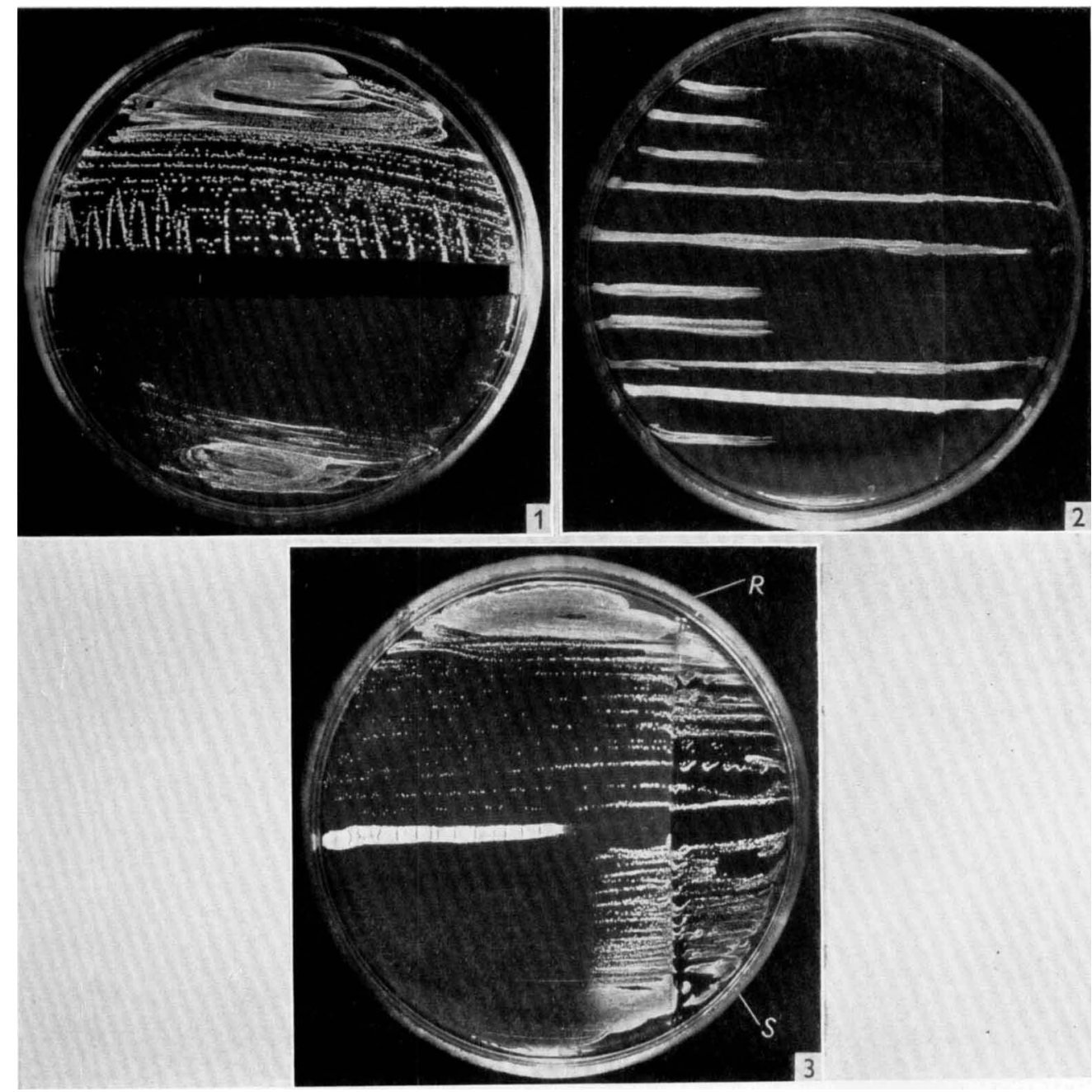

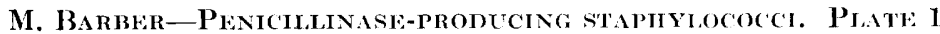

